\title{
A non-linear model for the dynamics of open cross-section thin-walled beams-Part II: forced motion
}

\author{
Angelo Di Egidio ${ }^{\mathrm{a}, *}$, Angelo Luongo ${ }^{\mathrm{a}}$, Fabrizio Vestroni ${ }^{\mathrm{b}}$
}

\author{
a Dipartimento di Ingegneria delle Strutture, Università di L'Aquila, delle Acque e del Terreno, Italy \\ 'Dipartimento di Ingegneria Strutturale e Geotecnica, Università di Roma "La Sapienza", Italy
}

\begin{abstract}
The discrete equations developed in Part I are here used to analyze the non-linear dynamics of an inextensional shear indeformable beam with given end constraints. The model takes into account the non-linear effects of warping and of torsional elongation. Non-linear 3D oscillations of a beam with a cross-section having one symmetry axis is examined. Only terms of higher magnitude are retained in the equations, which exhibit quadratic, cubic and combination resonances. A harmonic load acting in the direction of the symmetry axis and in resonance with the corresponding natural frequency, is considered. Steady-state solutions and their stability are studied; in particular the effects of non-linear warping and of torsional elongation on the response are highlighted.
\end{abstract}

Keywords: Beams; Open cross-section; Flexural-torsional dynamics; Non-linear resonances; Warping non-linear effects

\section{Introduction}

In Part I [1], the non-linear differential equations of motion of an inextensional shear undeformable beam with an open cross-section have been derived for the study of flexural-flexural-torsional interactions. The equations take into account the non-linear effects of warping and elongation of the longitudinal fibers due to torsion.

Interaction among flexural and torsional motion is studied in [2] in the case in which the torsional frequency of the beam is much higher than the bending frequencies. The torsional component can be statically condensed leading to two equations of motion in the two transversal displacement components. In [3], the non-planar responses of a cantilever beam, subject

\footnotetext{
${ }^{*}$ Corresponding author.
}

to lateral harmonic base-excitation, are investigated for the case of one-to-one internal resonances and a primary resonance, using two non-linear coupled integrodifferential equations of motion. As in [2], the torsional frequency of the beam is much higher than the bending frequencies. In beams with comparable flexural and torsional frequencies, torsional rotation should be added as an independent variable and at least three equations are necessary to describe the motion. Flexural-flexural-torsional interaction in a beam with comparable flexural and torsional frequencies is then considered in [4-7] analyzing non-linear coupling in free motion [4] and forced motion in internal resonance conditions $[5,6]$. The $3 \mathrm{D}$ dynamics of a cantilever beam with a compact section under a sinusoidal vertical force at its free end is analyzed in [7] in several internal resonance conditions involving flexural and torsional modes, by comparing experimental and analytical results. 
All cited works refer essentially to compact section beams where symmetry does not play any role, whereas in the present model open-cross-section beams, without any assumption of symmetry, are considered. The discrete form of the equations of motion obtained in Part I [1] is used to analyze the non-linear oscillations of a beam with a mono-symmetric cross-section. Due to the presence of many mixed terms, phenomena of modal coupling and internal resonance involving all the displacement components can occur, allowing the beam to undergo flexural-flexural-torsional vibrations. A beam has been considered having frequency ratios: $\omega_{1} / \omega_{3} \cong 2$ and $\omega_{2} / \omega_{3} \cong 3$, where $\omega_{1}$ is the flexural frequency in the symmetry direction and $\omega_{3}$ is the torsional frequency. These frequency ratios imply quadratic and cubic internal resonances and a combination resonance to occur.

A harmonic load acting in the direction of the symmetry axis, in an external resonance condition with flexural frequency, is considered. Multiple time scale method [8] is used to obtain modulation-phase equations and the reconstitution method first proposed in [9] is adopted to return to the true time domain. Steady-state solutions and their stability are studied by using the model proposed. The coefficients of the discrete equations of motion and some frequency-response curves are compared with those obtained by the model in which torsional elongation and non-linear warping are neglected, retaining linear Vlasov warping only.

\section{Amplitude and phase equations for the discrete model}

A beam with a monosymmetric cross-section, loaded by a distributed harmonic force applied to the beam's centroid axis acting along the section symmetric axis is considered. By assuming that the beam is inextensible and shear indeformable and using a three-mode discretization, the non-linear flexural-flexural-torsional oscillations are governed by the following three ordinary differential equations [1]:

$$
\begin{aligned}
\ddot{q}_{1}+d_{1} \dot{q}_{1}+k_{1} q_{1}= & c_{1} q_{2}^{2}+c_{2} q_{3}^{2}+c_{3} q_{2} q_{3}+c_{4} q_{1} q_{2}^{2} \\
& +c_{5} q_{1} q_{3}^{2}+c_{6} q_{1} q_{2} q_{3}+f_{1},
\end{aligned}
$$

$$
\begin{aligned}
\ddot{q}_{2}+d_{2} \dot{q}_{2}+k_{2} q_{2}= & c_{7} q_{1} q_{2}+c_{8} q_{1} q_{3}+c_{9} q_{2}^{3}+c_{10} q_{3}^{3} \\
& +c_{11} q_{1}^{2} q_{2}+c_{12} q_{1}^{2} q_{3}+c_{13} q_{2} q_{3}^{2} \\
& +c_{14} q_{2}^{2} q_{3}, \\
\ddot{q}_{3}+d_{3} \dot{q}_{3}+k_{3} q_{3}= & c_{15} q_{1} q_{2}+c_{16} q_{1} q_{3}+c_{17} q_{2}^{3} \\
& +c_{18} q_{3}^{3}+c_{19} q_{1}^{2} q_{2}+c_{20} q_{1}^{2} q_{3} \\
& +c_{21} q_{2} q_{3}^{2}+c_{22} q_{2}^{2} q_{3},
\end{aligned}
$$

where $q_{i}$ is the $i$ th mode amplitude, $d_{i}$ are the modal damping coefficients and $f_{1}(t)=p_{1} \mathrm{e}^{\mathrm{i} \Omega_{1} t}+$ c.c. is the load, of frequency $\Omega$, assumed to be in primary resonance with the $q_{1}$-mode. Moreover, the beam is assumed to be in internal resonance conditions of the kind $2: 3: 1$, so that quadratic, cubic and combination resonances occur.

The method of multiple time scales is employed to study the non-linear equations (1). Since non-linear terms are quadratic and cubic, a second-order expansion is developed. A small parameter $\varepsilon$ is introduced by ordering the linear damping and load amplitude as $d_{i}=\varepsilon^{2} \tilde{d}_{i} ; p_{i}=\varepsilon^{3} \tilde{p}_{i}$. Moreover, the displacements $q_{i}$ are expanded as

$$
\begin{aligned}
q_{i}\left(T_{0}, T_{1}, T_{2}, \varepsilon\right)= & \varepsilon q_{i}^{(0)}\left(T_{0}, T_{1}, T_{2}\right)+\varepsilon^{2} q_{i}^{(1)}\left(T_{0}, T_{1}, T_{2}\right) \\
& +\varepsilon^{3} q_{i}^{(2)}\left(T_{0}, T_{1}, T_{2}\right)+\cdots,
\end{aligned}
$$

where, $T_{0}=t, T_{1}=\varepsilon t, T_{2}=\varepsilon^{2} t$. $T_{0}$ is a fast scale, on which motions with frequencies of the order of $\Omega$ occur, while $T_{1}$ and $T_{2}$ are the slow scales, on which modulations of the amplitudes and phases take place. Substituting Eq. (2) into Eq. (1) and equating coefficients of like powers of $\varepsilon$, the following perturbation equations are obtained:

Order $\varepsilon$ :

$D_{00} q_{i}^{(0)}+\omega_{i}^{2} q_{i}^{(0)}=0, \quad(i=1,2,3)$.

Order $\varepsilon^{2}$ :

$$
\begin{aligned}
D_{00} q_{i}^{(1)}+\omega_{1}^{2} q_{1}^{(1)}= & -2 D_{01} q_{1}^{(0)}+c_{1} q_{2}^{(0)^{2}}+c_{2} q_{3}^{(0)^{3}} \\
& +c_{3} q_{2}^{(0)} q_{3}^{(0)}+\text { c.c., } \\
D_{00} q_{2}^{(1)}+\omega_{2}^{2} q_{2}^{(1)}= & -2 D_{01} q_{2}^{(0)}+c_{7} q_{1}^{(0)} q_{2}^{(0)} \\
& +c_{8} q_{1}^{(0)} q_{3}^{(0)}+\text { c.c. },
\end{aligned}
$$




$$
\begin{aligned}
D_{00} q_{3}^{(1)}+\omega_{3}^{*} q_{3}^{(1)}= & -2 D_{01} q_{3}^{(0)}+c_{15} q_{1}^{(0)} q_{2}^{(0)} \\
& +c_{16} q_{1}^{(0)} q_{3}^{(0)}+\text { c.c. }
\end{aligned}
$$

$\operatorname{Order} \varepsilon^{3}$ :

$$
\begin{aligned}
D_{00} q_{1}^{(2)} & +\omega_{1}^{2} q_{1}^{(2)} \\
= & -D_{11} q_{1}^{(0)}-2 D_{02} q_{1}^{(0)}-2 D_{01} q_{1}^{(1)} \\
& -d_{1} D_{0} q_{1}^{(0)}+2 c_{1} q_{2}^{(0)} q_{2}^{(1)} \\
& +2 c_{2} q_{3}^{(0)} q_{3}^{(1)}+c_{3} q_{2}^{(1)} q_{3}^{(0)}+c_{3} q_{2}^{(0)} q_{3}^{(1)} \\
& +c_{4} q_{1}^{(0)} q_{2}^{(0)^{2}}+c_{5} q_{1}^{(0)} q_{3}^{(0)^{2}} \\
& +c_{6} q_{1}^{(0)} q_{2}^{(0)} q_{3}^{(0)}+p_{1} \mathrm{e}^{\mathrm{i} \Omega_{1} T_{0}},
\end{aligned}
$$

$$
\begin{aligned}
D_{00} q_{2}^{(2)} & +\omega_{2}^{2} q_{2}^{(2)} \\
= & -D_{11} q_{2}^{(0)}-2 D_{02} q_{2}^{(0)}-2 D_{01} q_{2}^{(1)} \\
& -d_{2} D_{0} q_{2}^{(0)}+c_{7} q_{1}^{(1)} q_{2}^{(0)}+c_{7} q_{1}^{(0)} q_{2}^{(1)} \\
& +c_{8} q_{1}^{(1)} q_{3}^{(0)}+c_{8} q_{1}^{(0)} q_{3}^{(1)}+c_{9} q_{2}^{(0)^{3}} \\
& +c_{10} q_{3}^{(0)^{3}}+c_{11} q_{1}^{(0)^{2}} q_{2}^{(0)}+c_{12} q_{1}^{(0)^{2}} q_{3}^{(0)} \\
& +c_{13} q_{2}^{(0)} q_{3}^{(0)^{2}}+c_{14} q_{2}^{(0)^{2}} q_{3}^{(0)}
\end{aligned}
$$

$$
\begin{aligned}
D_{00} q_{3}^{(2)} & +\omega_{3}^{2} q_{3}^{(2)} \\
= & -D_{11} q_{3}^{(0)}-2 D_{02} q_{3}^{(0)}-2 D_{01} q_{3}^{(1)} \\
& -d_{3} D_{0} q_{3}^{(0)}+c_{15} q_{1}^{(1)} q_{2}^{(0)}+c_{15} q_{1}^{(0)} q_{2}^{(1)} \\
& +c_{16} q_{1}^{(1)} q_{3}^{(0)}+c_{16} q_{1}^{(0)} q_{3}^{(1)}+c_{17} q_{2}^{(0)^{3}} \\
& +c_{18} q_{3}^{(0)^{3}}+c_{19} q_{1}^{(0)^{2}} q_{2}^{(0)}+c_{20} q_{1}^{(0)^{2}} q_{3}^{(0)} \\
& +c_{21} q_{2}^{(0)} q_{3}^{(0)^{2}}+c_{22} q_{2}^{(0)^{2}} q_{3}^{(0)}
\end{aligned}
$$

where $D_{i}()=\partial() / \partial T_{i}, D_{i j}()=\partial^{2}() / \partial T_{i} \partial T_{j}(i, j=0,1,2)$ and the tilde has been omitted for simplicity. The solution to the first-order perturbation equations (3) is

$q_{i}^{(0)}=A_{i}\left(T_{1}, T_{2}\right) \mathrm{e}^{\mathrm{i} \omega_{i} T_{0}}+$ c.c.

In order to investigate the system response under internal and external resonance conditions, three detuning parameters $\sigma_{i}$ are introduced

$$
\begin{aligned}
& \Omega_{1}=\omega_{1}+\varepsilon^{2} \sigma_{1}, \\
& 2 \omega_{3}=\omega_{1}+\varepsilon \sigma_{2}, \\
& 3 \omega_{3}=\omega_{2}+\varepsilon^{2} \sigma_{3} .
\end{aligned}
$$

The first relation accounts for the primary external resonance, while the last two relations account for the quadratic and the cubic internal resonances, respectively. Substituting Eq. (6) into Eqs. (4) and zeroing secular terms, it follows that

$$
\begin{aligned}
D_{1} A_{1}= & -2 \mathrm{i} \alpha_{1} A_{3}^{2} \mathrm{e}^{\mathrm{i} \sigma_{2} T_{1}}-2 \mathrm{i} \alpha_{2} A_{2} \bar{A}_{3} \mathrm{e}^{\mathrm{i}\left(\sigma_{2} T_{1}-\sigma_{3} T_{2}\right)}, \\
D_{1} A_{2}= & -2 \mathrm{i} \alpha_{3} A_{1} A_{3} \mathrm{e}^{\mathrm{i}\left(\sigma_{3} T_{2}-\sigma_{2} T_{1}\right)}, \\
D_{1} A_{3}= & -2 \mathrm{i} \alpha_{4} \bar{A}_{1} A_{2} \mathrm{e}^{\mathrm{i}\left(\sigma_{2} T_{1}-\sigma_{3} T_{2}\right)} \\
& -2 \mathrm{i} \alpha_{5} A_{1} \bar{A}_{3} \mathrm{e}^{-\mathrm{i} \sigma_{2} T_{1}},
\end{aligned}
$$

where coefficients $\alpha_{i}$ are reported in the appendix. The solution to the $\varepsilon^{2}$-order perturbation equations reads:

$$
\begin{aligned}
q_{1}^{(1)}= & K_{1} c_{1} A_{2}^{2} \mathrm{e}^{\mathrm{i} 2 \omega_{2} T_{0}}+K_{2} c_{3} A_{2} A_{3} \mathrm{e}^{\mathrm{i}\left(\omega_{2}+\omega_{3}\right) T_{0}} \\
& +\frac{c_{1}}{\omega_{1}^{2}} A_{2} \bar{A}_{2}+\frac{c_{2}}{\omega_{1}^{2}} A_{3} \bar{A}_{3}+\text { c.c. } \\
q_{2}^{(1)}= & K_{3} c_{7} A_{1} A_{2} \mathrm{e}^{\mathrm{i}\left(\omega_{1}+\omega_{2}\right) T_{0}}+K_{4} c_{7} \bar{A}_{1} A_{2} \mathrm{e}^{\mathrm{i}\left(\omega_{2}-\omega_{1}\right) T_{0}} \\
& +K_{5} c_{8} \bar{A}_{1} A_{3} \mathrm{e}^{\mathrm{i}\left(\omega_{3}-\omega_{1}\right) T_{0}}+\text { c.c. } \\
q_{3}^{(1)}= & K_{6} c_{15} A_{1} A_{2} \mathrm{e}^{\mathrm{i}\left(\omega_{1}+\omega_{2}\right) T_{0}} \\
& +K_{7} c_{16} A_{1} A_{3} \mathrm{e}^{\mathrm{i}\left(\omega_{1}+\omega_{3}\right) T_{0}}+\text { c.c. },
\end{aligned}
$$

where coefficients $K_{i}$ are reported in the appendix. Eqs. (6) and (9) are substituted into Eqs. (5); zeroing again secular terms and taking into account Eqs. (7), leads to

$$
\begin{aligned}
D_{2} A_{1}= & \left(-\mathrm{i} d_{1} \omega_{1} A_{1}+p_{1} \mathrm{e}^{\mathrm{i} \sigma_{1} T_{2}}+b_{1} \bar{A}_{1} A_{2} A_{3} \mathrm{e}^{\mathrm{i}\left(2 \sigma_{2} T_{1}-\sigma_{3} T_{2}\right)}\right. \\
& +b_{2} A_{1} A_{3} \bar{A}_{3}+b_{3} A_{3}^{2} \mathrm{e}^{\mathrm{i} \sigma_{2} T_{1}}+b_{4} A_{1} A_{2} \bar{A}_{2} \\
& \left.+b_{5} A_{2} \bar{A}_{3} \mathrm{e}^{\mathrm{i}\left(\sigma_{2} T_{1}-\sigma_{3} T_{2}\right)}\right) / 2 \mathrm{i} \omega_{1},
\end{aligned}
$$

$$
\begin{aligned}
D_{2} A_{2}= & \left(-\mathrm{i} d_{2} \omega_{2} A_{2}+b_{6} A_{3}^{3} \mathrm{e}^{\mathrm{i} \sigma_{3} T_{2}}+b_{7} A_{2} A_{3} \bar{A}_{3}\right. \\
& +b_{8} A_{1} \bar{A}_{1} A_{2}+b_{9} A_{1}^{2} \bar{A}_{3} \mathrm{e}^{\mathrm{i}\left(\sigma_{2} T_{1}-2 \sigma_{3} T_{2}\right)}+b_{10} A_{2}^{2} \bar{A}_{2} \\
& \left.+b_{11} A_{1} A_{3} \mathrm{e}^{\mathrm{i}\left(\sigma_{3} T_{2}-\sigma_{2} T_{1}\right)}\right) / 2 \mathrm{i} \omega_{2},
\end{aligned}
$$




$$
\begin{aligned}
D_{2} A_{3}= & \left(\mathrm{i} d_{3} \omega_{3} A_{3}+b_{12} A_{2} \bar{A}_{3}^{2} \mathrm{e}^{-\mathrm{i} \sigma_{3} T_{2}}+b_{13} A_{2} \bar{A}_{2} A_{3}\right. \\
& +b_{14} A_{1} \bar{A}_{1} A_{3}+b_{15} \bar{A}_{1} A_{2} \mathrm{e}^{\mathrm{i}\left(\sigma_{2} T_{1}-\sigma_{3} T_{2}\right)} \\
& +b_{16} A_{3}^{2} \bar{A}_{3}+b_{17} A_{1}^{2} \bar{A}_{2} \mathrm{e}^{\mathrm{i}\left(\sigma_{3} T_{2}-2 \sigma_{2} T_{1}\right)} \\
& \left.+b_{18} A_{1} \bar{A}_{3} \mathrm{e}^{-\mathrm{i} \sigma_{2} T_{1}}\right) / 2 \mathrm{i} \omega_{3}
\end{aligned}
$$

where coefficients $b_{i}$ are given in the appendix.

Eqs. (8) and (10) are rewritten in terms of polar quantities by defining

$A_{j}=\frac{1}{2} a_{j} \mathrm{e}^{\mathrm{i} \psi_{j}}$,

where $a_{i}$ are real amplitudes and $\psi_{i}$ are the phases. In the previous Eqs. (8) and (10), the explicit dependence on time is eliminated by introducing the following phase-differences:

$\gamma_{1}=\psi_{1}-\sigma_{1} T_{2}$

$\gamma_{2}=2 \psi_{3}-\psi_{1}+\sigma_{2} T_{1}$,

$\gamma_{3}=3 \psi_{3}-\psi_{2}+\sigma_{3} T_{2}$.

The reconstitution method first proposed in [9] is adopted to return to true time $t$, according to the following rules:

$\dot{a}_{i}=\varepsilon D_{1} a_{i}+\varepsilon^{2} D_{2} a_{i}$,

$\dot{\vartheta}_{i}=\varepsilon D_{1} \vartheta_{i}+\varepsilon^{2} D_{2} \vartheta_{i}$.

Using Eqs. (8) and (10) and taking into account Eqs. (11) and (12), the following amplitude and phase equations are finally obtained:

$$
\begin{aligned}
\dot{a}_{1}= & -d_{1}^{*} a_{1}+h_{1} a_{3}^{2} \sin \gamma_{2}+h_{2} a_{2} a_{3} \sin \left(\gamma_{2}-\gamma_{3}\right) \\
& +h_{3} a_{1} a_{2} a_{3} \sin \left(2 \gamma_{2}-\gamma_{3}\right)-f_{1}^{*} \sin \gamma_{1}, \\
\dot{a}_{2}= & -d_{2}^{*} a_{2}+h_{4} a_{1} a_{3} \sin \left(\gamma_{3}-\gamma_{2}\right)+h_{5} a_{3}^{3} \sin \gamma_{3} \\
& +h_{6} a_{1}^{2} a_{3} \sin \left(\gamma_{3}-2 \gamma_{2}\right), \\
\dot{a}_{3}= & -d_{3}^{*} a_{3}-h_{7} a_{1} a_{3} \sin \gamma_{2}+h_{8} a_{1} a_{2} \sin \left(\gamma_{2}-\gamma_{3}\right) \\
& -h_{9} a_{2} a_{3}^{2} \sin \gamma_{3}+h_{10} a_{1}^{2} a_{2} \sin \left(\gamma_{3}-2 \gamma_{2}\right), \\
a_{1} \dot{\gamma}_{1}= & -h_{1} a_{3}^{2} \cos \gamma_{2}-h_{2} a_{2} a_{3} \cos \left(\gamma_{2}-\gamma_{3}\right) \\
& -h c_{3} a_{1} a_{2} a_{3} \cos \left(2 \gamma_{2}-\gamma_{3}\right)-h_{14} a_{1} a_{3}^{2} \\
& -h_{15} a_{1} a_{2}^{2}-f_{1}^{*} \cos \gamma_{1}-\sigma_{1} a_{1},
\end{aligned}
$$

$$
\begin{aligned}
a_{1} a_{3} \dot{\gamma}_{2}= & \left(h_{1} a_{3}^{3}-2 h_{7} a_{1}^{2} a_{3}\right) \cos \gamma_{2} \\
& +\left(h_{2} a_{2} a_{3}^{2}-2 h_{8} a_{1}^{2} a_{2}\right) \cos \left(\gamma_{2}-\gamma_{3}\right) \\
& -2 h_{9} a_{1} a_{2} a_{3}^{2} \cos \gamma_{3}+\left(h_{3} a_{1} a_{2} a_{3}^{2}-2 h_{10} a_{1}^{3} a_{2}\right) \\
& \times \cos \left(\gamma_{3}-2 \gamma_{2}\right)-2 h_{11} a_{1} a_{2}^{2} a_{3}-2 h_{14} a_{1}^{3} a_{3} \\
& -2 h_{13} a_{1} a_{3}^{3}+h_{14} a_{1} a_{3}^{3}+h_{15} a_{1} a_{2}^{2} a_{3} \\
& +f_{1}^{*} a_{3} \cos \gamma_{1}+\sigma_{2} a_{1} a_{3}, \\
a_{2} a_{3} \dot{\gamma}_{3}= & -3 h_{7} a_{1} a_{2} a_{3} \cos \gamma_{2}+\left(h_{4} a_{1} a_{3}^{2}-3 h_{8} a_{1} a_{2}^{2}\right) \\
& \times \cos \left(\gamma_{2}-\gamma_{3}\right)+\left(h_{5} a_{3}^{4}-3 h_{9} a_{2}^{2} a_{3}^{2}\right) \cos \gamma_{3} \\
& +\left(h_{6} a_{1}^{2} a_{3}^{2}-3 h_{10} a_{1}^{2} a_{2}^{2}\right) \cos \left(\gamma_{3}-2 \gamma_{2}\right) \\
& -3 h_{11} a_{2}^{3} a_{3}-3 h_{14} a_{1}^{2} a_{2} a_{3}-3 h_{13} a_{2} a_{3}^{3} \\
& +h_{16} a_{2} a_{3}^{3}+h_{17} a_{1}^{2} a_{2} a_{3}+h_{18} a_{2}^{3} a_{3}+\sigma_{3} a_{2} a_{3},
\end{aligned}
$$

where coefficients $h_{i}, f_{1}^{*}, d_{i}^{*}$ are reported in the appendix. Eqs. (14) describe the non-linear resonant motion of the system; the steady-state solutions and their stability are studied for a particular mechanical system in the next section.

\section{Numerical results}

\subsection{Sample mechanical system}

Non-linear coupling and resonant motions are investigated for a beam simply supported at the ends and restrained by torsional constraints. Warping at the end sections is admitted and only one of the supports is free to move in the longitudinal direction. The boundary conditions at $z=0, l$ are

$$
\begin{aligned}
& \left\{\begin{array} { l } 
{ u _ { 1 } ( 0 ) = 0 , } \\
{ u _ { 2 } ( 0 ) = 0 , } \\
{ \vartheta _ { 3 } ( 0 ) = 0 , }
\end{array} \quad \left\{\begin{array}{l}
u_{1}(l)=0, \\
u_{2}(l)=0, \\
\vartheta_{3}(l)=0,
\end{array}\right.\right. \\
& \left\{\begin{array} { l } 
{ E I _ { 2 } u _ { 1 } ^ { \prime \prime } ( 0 ) = 0 , } \\
{ E I _ { 1 } u _ { 2 } ^ { \prime \prime } ( 0 ) = 0 , } \\
{ E \Gamma \vartheta _ { 3 } ^ { \prime \prime } ( 0 ) = 0 , }
\end{array} \quad \left\{\begin{array}{l}
E I_{2} u_{1}^{\prime \prime}(l)=0, \\
E I_{1} u_{2}^{\prime \prime}(l)=0, \\
E \Gamma \vartheta_{3}^{\prime \prime}(l)=0 .
\end{array}\right.\right.
\end{aligned}
$$

They are such that simple closed-form expressions for the eigenvalues and the eigenfunctions of the linearized system can be obtained. 


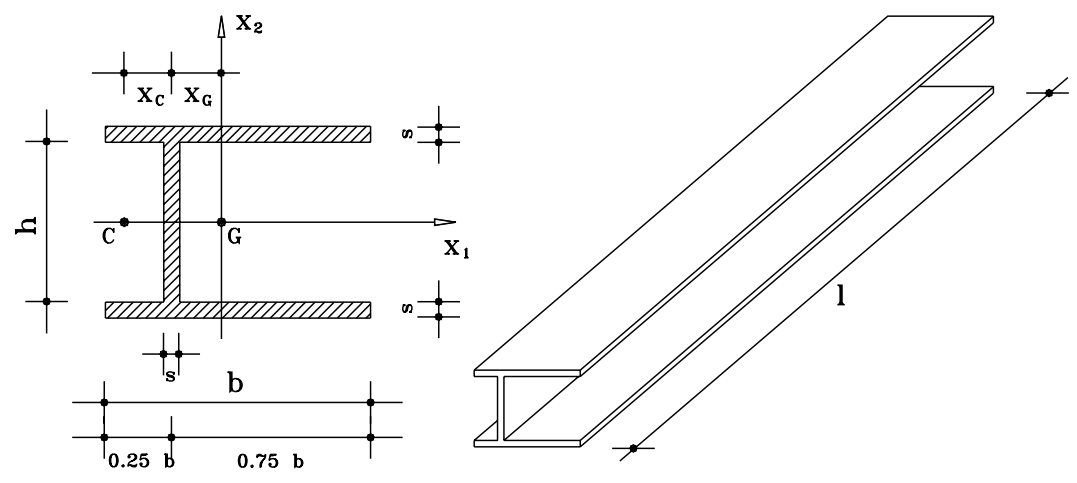

Fig. 1. Geometrical beam characteristics.

The cross-section of the beam is illustrated in Fig. 1. It has a symmetry axis $x_{1}$; moreover the abscissas of the shear center $x_{\mathrm{C}}$ and of the centroid $x_{\mathrm{G}}$ are equal to:

$x_{\mathrm{C}}=\frac{3 s^{2} h^{2} b^{2}}{12 b s^{2} h^{2}+8 s^{2} h^{3}}, \quad x_{\mathrm{G}}=\frac{b^{2} s}{2 s(2 b+h)}$.

The aspect ratios are assumed to be $s / h \cong 1 / 25$, $h / b \cong 3 / 5, h / l \cong 1 / 48$, for which the internal resonance conditions $(7)_{2},(7)_{3}$ are satisfied. In the numerical investigation the following geometrical and material characteristic are used: $h=3.0 \mathrm{~cm}, b=5.0 \mathrm{~cm}$, $s=0.12 \mathrm{~cm}, l=144 \mathrm{~cm}, E=1,22,0000 \mathrm{~kg} / \mathrm{cm}^{2}, G=$ $469,230 \mathrm{~kg} / \mathrm{cm}^{2}, \rho=9.1 \times 10^{-6} \mathrm{~kg}_{m} / \mathrm{cm}^{3}$. The dimensions of the section of the beam have been chosen in such a way as to make possible experimental investigations in future; the material considered is copper.

The solution of the linear free dynamic problem furnishes the following first three eigenvalues and eigenfunctions:

$$
\begin{aligned}
\omega_{1} & =235.40 \mathrm{rad} / \mathrm{s}, \\
\omega_{2} & =354.20 \mathrm{rad} / \mathrm{s}, \\
\omega_{3} & =117.76 \mathrm{rad} / \mathrm{s} \\
\Phi_{1} & =\left(\begin{array}{l}
1 \\
0 \\
0
\end{array}\right) \sin \frac{\pi z}{l}, \\
\Phi_{2} & =\left(\begin{array}{c}
0 \\
-0.958813 \\
0.284039
\end{array}\right) \sin \frac{\pi z}{l},
\end{aligned}
$$

$$
\Phi_{3}=\left(\begin{array}{c}
0 \\
0.499159 \\
0.866511
\end{array}\right) \sin \frac{\pi z}{l} .
$$

The displacement components $u_{1}(z, t), u_{2}(z, t)$, $\vartheta_{3}(z, t)$ are obtained from the modal amplitudes $q_{1}(t)$ by the following relations:

$$
\begin{aligned}
u_{1}= & \varphi_{11} a_{1} \cos \left(\omega_{1} t+\psi_{1}\right) \sin \left(\frac{\pi z}{l}\right), \\
u_{2}= & {\left[\varphi_{22} a_{2} \cos \left(\omega_{2} t+\psi_{2}\right)\right.} \\
& \left.+\varphi_{23} a_{3} \cos \left(\omega_{3} t+\psi_{3}\right)\right] \sin \left(\frac{\pi z}{l}\right), \\
\vartheta_{3}= & {\left[\varphi_{32} a_{2} \cos \left(\omega_{2} t+\psi_{2}\right)\right.} \\
& \left.+\varphi_{33} a_{3} \cos \left(\omega_{3} t+\psi_{3}\right)\right] \sin \left(\frac{\pi z}{l}\right),
\end{aligned}
$$

where $\varphi_{i j}$ is the $j$ th component of the $i$ th eigenfunction $\Phi_{i}$. According to Part I of this work, the eigenfunctions $\Phi_{i}$ are used as shape functions to discretize the equations of motion through the Galerkin procedure. The displacements of the midspan cross-section in the first three modes are illustrated in Fig. 2. Due to the symmetry, the displacement component $u_{1}$ is uncoupled from the other two.

The coefficients of the discretized equations of motion (1), are then evaluated in non-dimensional form, as illustrated in Part I, for a beam in which non-linear warping and torsional elongation of the longitudinal fibers are accounted (Model 1) and also for the case in which these contributions are not taken into account and only the linear Vlasov contribution to warping is considered (Model 2). 

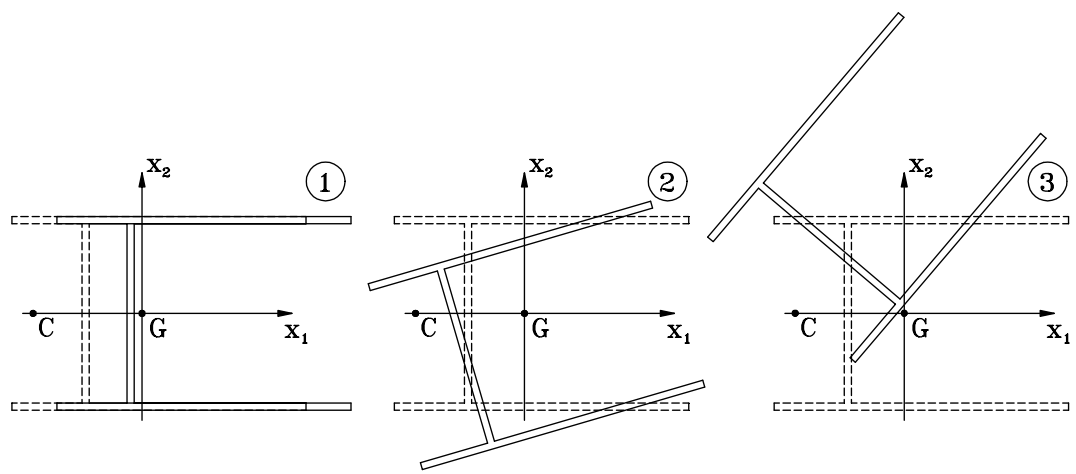

Fig. 2. Midspan section displacements in the first three eigenfunctions.

Table 1

Coefficients of the non-dimensional discretized equations of motion

\begin{tabular}{lllllllll}
\hline Eq. (1) & $c_{1}$ & $c_{2}$ & $c_{3}$ & $c_{4}$ & $c_{5}$ & $c_{6}$ & & \\
\hline Model 1 & -4.2408 & -5.3393 & -9.5418 & -18.0918 & -26.3292 & -43.6505 & & \\
Model 2 & -0.4896 & 0.1199 & -0.4905 & -18.0918 & -26.3296 & -43.6505 & & \\
\hline Eq. (2) & $c_{7}$ & $c_{8}$ & $c_{9}$ & $c_{10}$ & $c_{11}$ & $c_{12}$ & $c_{13}$ & $c_{14}$ \\
\hline Model 1 & -61.4079 & -70.1305 & -212.885 & -1132.10 & -103.679 & -125.075 & -1633.40 & -319.262 \\
Model 2 & -5.6042 & -2.8110 & -66.7693 & -8.2102 & -103.679 & -125.075 & 17.2263 & 100.4690 \\
\hline Eq. (3) & $c_{15}$ & $c_{16}$ & $c_{17}$ & $c_{18}$ & $c_{19}$ & $c_{20}$ & $c_{21}$ & $c_{22}$ \\
\hline Model 1 & -24.728 & -27.676 & -127.672 & -829.180 & -56.5657 & -68.2389 & -1596.67 & -907.797 \\
Model 2 & -1.2713 & 0.6214 & 15.1459 & 1.8149 & -56.5657 & -68.2389 & -11.1393 & 7.7907 \\
\hline
\end{tabular}

The values of the coefficients for the two model are listed in Table 1. It is noticed that the new contributions notably change the values of many coefficients. In particular, several resonant terms are strongly modified by these effects, thus implying the motion is strongly affected by them. Accordingly, a remarkable change is registered in most of coefficients of the modulation equations (14), as shown in Table 2 . This confirms that the motion will change very much due to the contribution of warping and torsional elongation.

\subsection{Steady-state motions and stability}

Steady-state solutions are determined by zeroing the right-hand members of the modulation equations (14) and solving the non-linear system. Stability analysis is then performed by analyzing the eigenvalues of the Jacobian matrix of the non-linear equations cal- culated at the fixed points. Most results, which are discussed in the following, are relevant to the Model 1; a few numerical investigation has been performed also with Model 2, although it is inaccurate for the open cross-section beams, just to point out the modifications in the response produced by the refined presented theory.

Amplitude-load curves are reported in Fig. 3 for Model 1. They have been obtained for external forces in a perfect resonance condition $\left(\sigma_{1}=0\right)$ and for two values of damping (Figs. 3a and b). The smaller damping is first considered (Fig. 3a). A unimodal linear solution branch, $a_{1} \neq 0, a_{2}=a_{3}=0$, is found. On this branch, at a point very near to the axes origin, one three-modal solution branch bifurcates. Due to the presence of some saddle-nodes, the three-modal solution branch alternatively loses and regains stability. However, this behavior appears at very high values 
Table 2

Coefficients of the modulation equations of motion in the two Models 1 and 2

\begin{tabular}{lllllll}
\hline & $h_{1}$ & $h_{2}$ & $h_{3}$ & $h_{4}$ & $h_{5}$ & $h_{6}$ \\
\hline Model 1 & -1.3362 & -2.3878 & 68.4789 & -11.6907 & -89.2138 & 223.722 \\
Model 2 & 0.0299 & -0.1227 & -5.1118 & -0.4686 & -0.6895 & -9.8221 \\
\hline & $h_{7}$ & $h_{8}$ & $h_{9}$ & $h_{10}$ & $h_{11}$ & $h_{12}$ \\
\hline Model 1 & -13.8381 & -12.4138 & -383.395 & -4.1440 & -445.381 & 40.0938 \\
Model 2 & 0.3107 & -0.6382 & -2.8095 & -13.0776 & 3.6203 & -33.5854 \\
\hline & $h_{13}$ & $h_{14}$ & $h_{15}$ & $h_{16}$ & $h_{17}$ & $h_{18}$ \\
\hline Model 1 & -530.551 & 49.3155 & -15.2101 & -227.127 & 85.7115 & 93.9876 \\
Model 2 & 1.4106 & -6.4672 & -4.4475 & 2.7422 & -16.5887 & 17.1358 \\
\hline
\end{tabular}
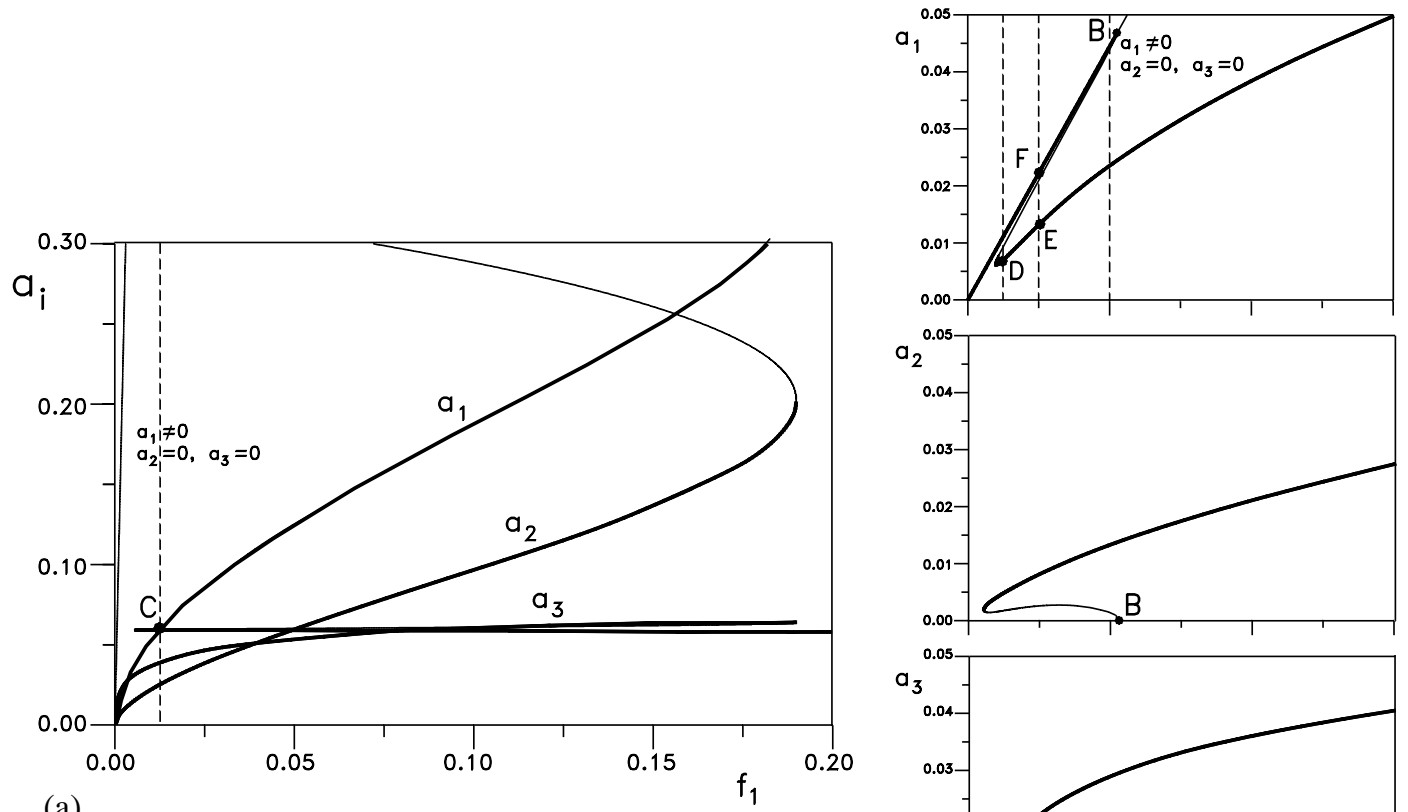

(a)

(b)
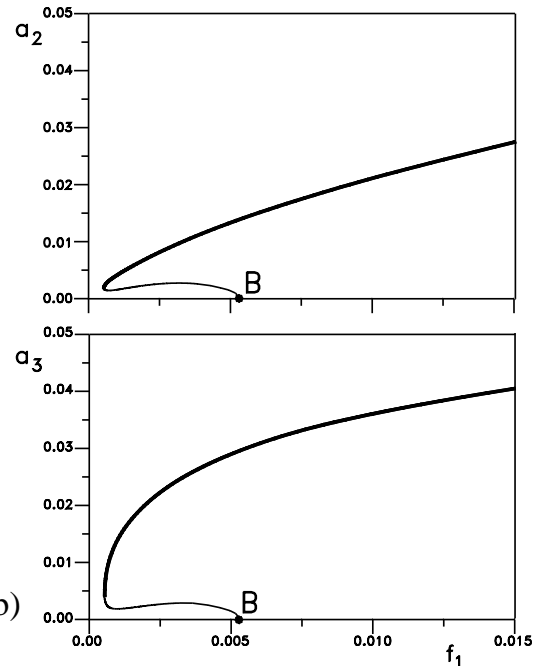

Fig. 3. Model 1, amplitude-load curves: (a) $d=0.01$; (b) $d=0.075$. Perfect external resonance: $\sigma_{1}=0$. Thick line: stable solutions; thin line: unstable solutions.

of the displacements at which the validity of the performed analysis is in doubt. By limiting the attention to moderately large displacements, the amplitudes curves shown in Fig. $3 \mathrm{~b}$ are obtained for a higher damping to better observe the kind of bifurcation, since point $B$ goes away from the beginning of the unimodal branch, as damping is increased. In general, the angular coefficient of the unimodal solution and the position of 

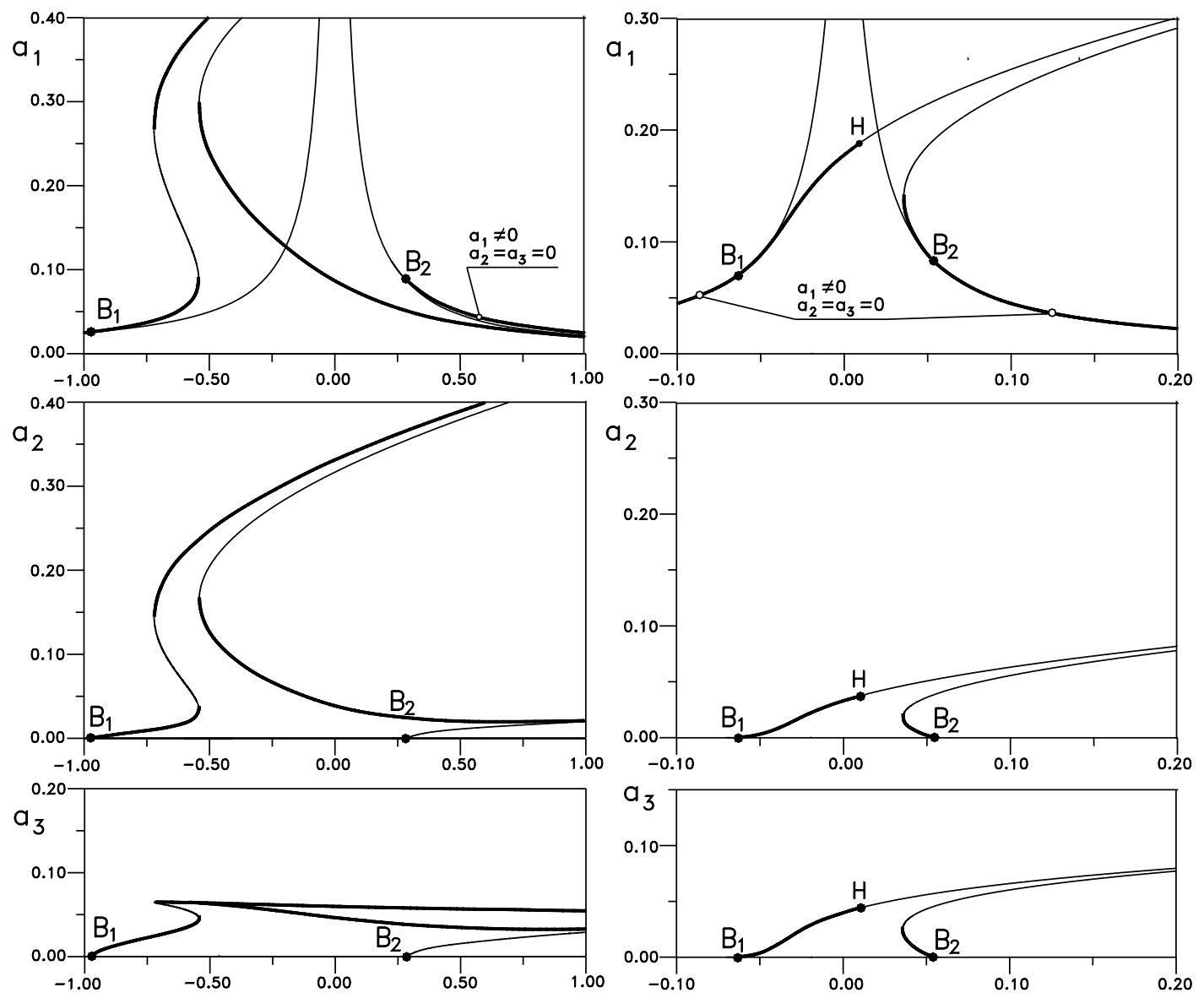

(a)

$\sigma_{1}$

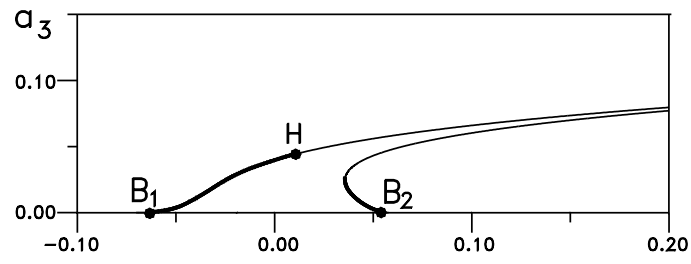

(b)

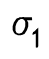

Fig. 4. Model 1, frequency-response curves: (a) $f_{1}=0.025$; (b) $f_{1}=0.0045$. Damping value: $d=0.01$. Thick line: stable solutions; thin line: unstable solutions.

the bifurcation point depend on damping. At $B$, the $a_{1}$ bifurcated branch is tangent to the unimodal solution and is unstable. After $B$, the unimodal oscillation is unstable, which means that unimodal oscillation is practically always unstable. It is worth noting that the bifurcated three component solution has a stable branch which starts very close to the origin, that is for very small value of the force.

Frequency-response curves are then analyzed in Fig. 4, in which two different loads are considered. The unimodal solution becomes unstable at two points $B_{1}$ and $B_{2}$, located on opposite sides with respect to the resonance. Consequently, the unimodal solution is unstable almost everywhere, even with respect to the parameter $\sigma_{1}$, apart from a small range of $f_{1}$, as already observed in Fig. 3. The branch bifurcating from $B_{1}$ is initially stable for the two loads considered, while the branch bifurcating from $B_{2}$ is initially unstable for the higher load (Fig. 4a) and stable for the lower load (Fig. 4b). However, both change stability at higher amplitudes, due to the occurrence of saddle-nodes in Fig. 4a, or Hopf bifurcations $H$ and saddle-nodes in Fig. 4b. It is interesting to note that a saturation phenomenon of $a_{3}$ component occurs which is manifest in the amplitude-load curve (Fig. 3a) and in the frequency-response curve (Fig. 4a).

The results furnished by Model 1 differ notably from those obtained omitting the contribution of non-linear 

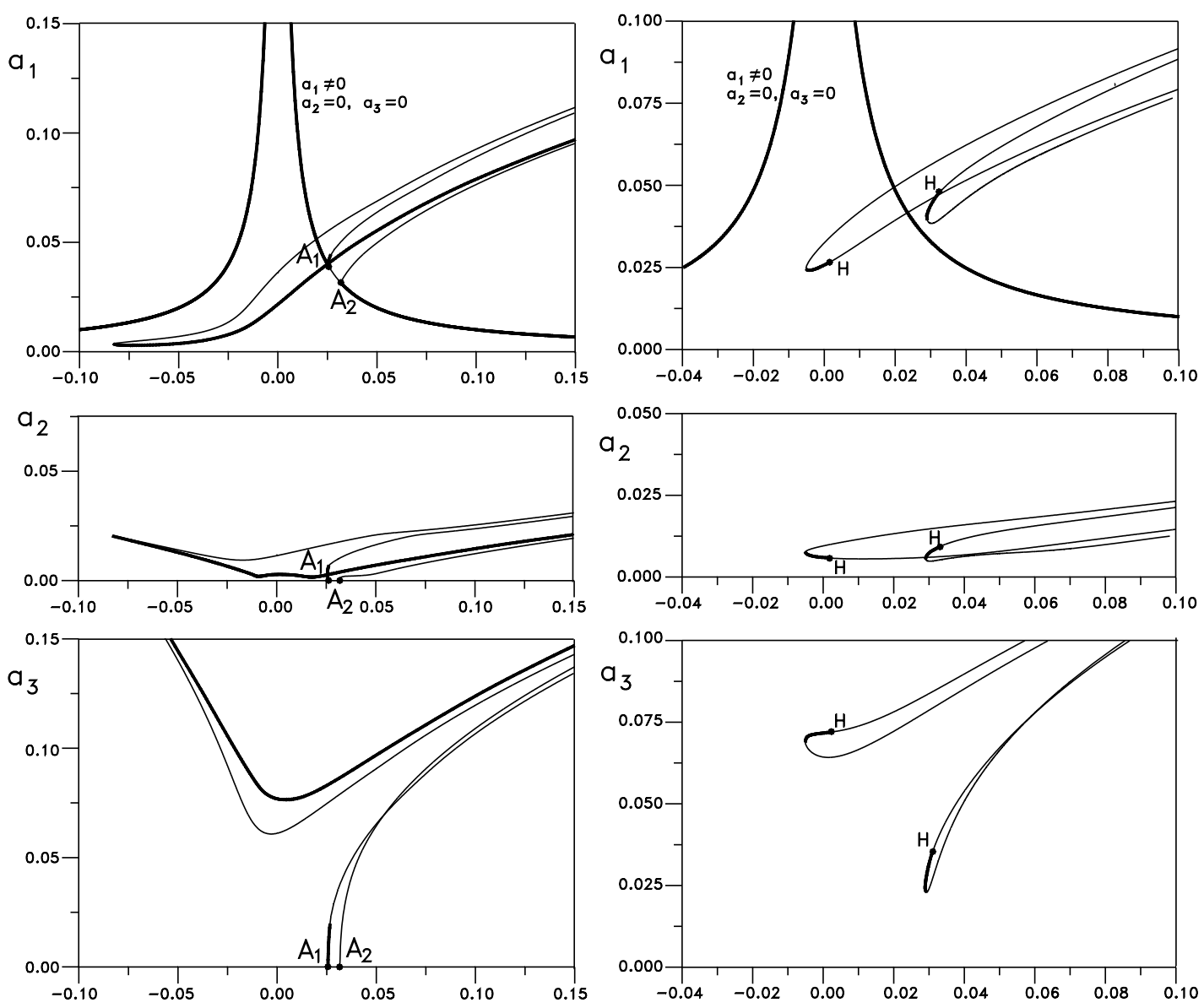

(a)

$\sigma_{1}$

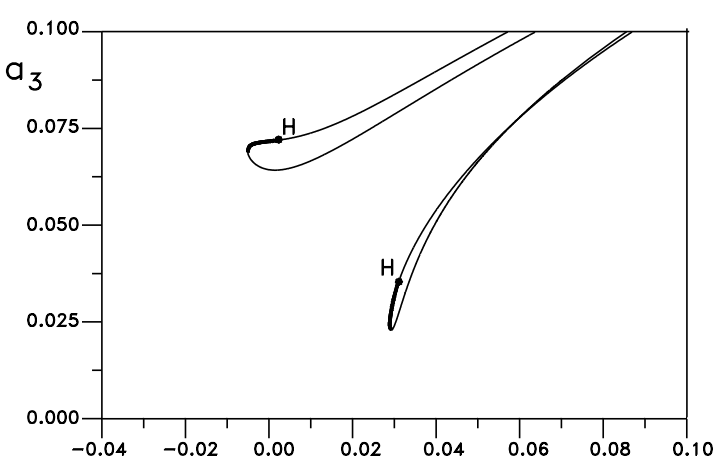

(b)

$\sigma_{1}$

Fig. 5. Model 2, frequency-response curves: (a) $d=0.0005$; (b) $d=0.01$ Load value: $f_{1}=0.001$. Thick line: stable solutions; thin line: unstable solutions.

warping and torsional elongation of the longitudinal fibers (Model 2). The differences can be appreciated by comparing the frequency-response curves of Fig. 4 with those obtained with Model 2, reported in Fig. 5. For small damping $d$ (Fig. 5a) on the unimodal linear solution branch, $a_{1} \neq 0, a_{2}=a_{3}=0$, two bifurcation points $A_{1}$ and $A_{2}$ occur. Their distance depends on the damping, but they are in any case very close. As a consequence, in contrast with Model 1, the unimodal solution is stable everywhere, except for the small zone between the two bifurcation points. Two three-modal solution branches bifurcate from the points $A_{1}$ and $A_{2}$, but only one branch is stable; this is characterized by a $a_{3}$ modal amplitude much greater than the other components. No saturation phenomenon of the third component is observed. Increasing the damping $d$, the two bifurcation points first coalesce and then bifurcation disappears (see Fig. 5b). In this case, the unimodal solutions are always stable, while the three-modal solution curve is unstable almost everywhere due to a saddle-node and a Hopf bifurcation at $H$. The beam oscillates indefinitely in the plane of the excitation, while Model 1 foresees an oscillation of the beam excited by a force in the symmetry plane characterized by comparable out-of-plane components. 


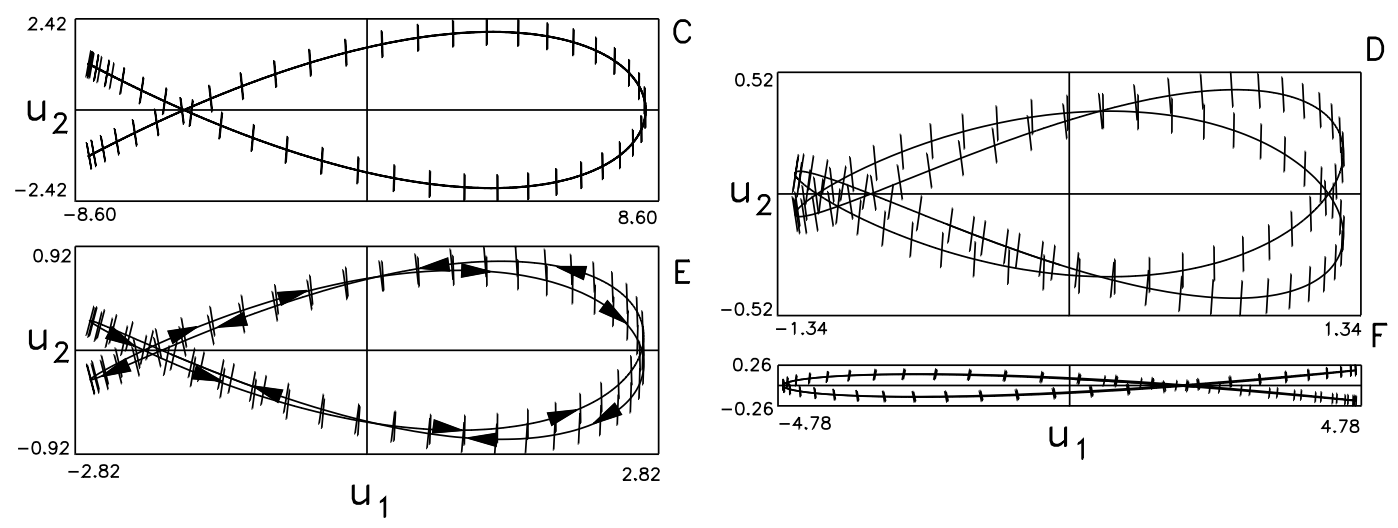

Fig. 6. Model 1, orbits of beam middle section: (a) $d=0.01$; (b) $d=0.075$.

Some orbits of the displacements and rotation of the beam middle-section are illustrated in Fig. 6 at the points marked in Figs. 3a and b. Orbits of the stable and unstable solutions always keep a similar pattern along the branch. Due to Eqs. (18), the displacement components $u_{2}, \vartheta_{3}$ oscillate with frequencies $\omega_{2}$ and $\omega_{3}$. One of these frequencies can be more important than the other in the displacement components $u_{2}$ and $\vartheta_{3}$ evolution. If $\varphi_{i 2} a_{2}$ term is $>\varphi_{i 3} a_{3}$ term $(i=2,3)$ in Eqs. (18), $\omega_{2}$ is the prevailing frequency in the evolution of the displacement components $u_{2}$ and $\vartheta_{3}$; on the contrary, $\omega_{3}$ becomes the prevailing frequency. The running direction of the orbits is reported also when useful. The patterns of the orbits presented are quite different from those obtained by Model 2 which are not reported for the sake of brevity.

\section{Conclusions}

The non-linear oscillations of a supported beam have been investigated in Part II of this paper by using the ODE's obtained in Part I [1]. They have been specialized for a beam having one symmetry axis. The beam is in internal resonance conditions and is excited by a force in external resonance condition with the first mode, along the symmetry axis. Amplitude and phase equations have been obtained through multiple scale method and solved numerically. For the beam model, in which the effects due to non-linear warping and torsional elongation of the longitudinal fibers are taken into account (Model 1), the behavior is illustrated by frequency-response and amplitude-load curves, evaluated for different values of damping and load. Some results are compared with those obtained by neglecting the non-linear warping and torsional elongation of the longitudinal fibers (Model 2).

The results have shown that warping and torsional elongation produce deep modifications in the dynamic behavior of the beam. In fact, the internal resonance conditions considered interest those terms of the discretized equations of motion, that are more strongly modified by the effects taken into account in the model. These latter consequently produce strong modifications in the behavior of the system; quantitative and qualitative differences are evidenced, these latter affecting the stability of the oscillations. However, the case analyzed here should be considered as a limit case, in which the influence of the non-linear warping and torsional elongation effects is magnified.

\section{Acknowledgements}

This work has been partially supported by the funds of MURST (Ministry of University, Scientific and Technological Research) under the Grant COFIN 9798, 99-00. 
Appendix

Coefficients $\alpha_{i}$ of Eqs. (8) are

$\alpha_{1}=\frac{c_{2}}{4 \omega_{1}}, \quad \alpha_{2}=\frac{c_{3}}{4 \omega_{1}}, \quad \alpha_{3}=\frac{c_{8}}{4 \omega_{2}}$,

$\alpha_{4}=\frac{c_{15}}{4 \omega_{3}}, \quad \alpha_{5}=\frac{c_{16}}{4 \omega_{3}}$.

Coefficients $K_{i}$ of Eqs. (9) are

$$
\begin{aligned}
& K_{1}=\frac{1}{\omega_{1}^{2}-\left(2 \omega_{2}\right)^{2}}, \quad K_{2}=\frac{1}{\omega_{1}^{2}-\left(\omega_{2}+\omega_{3}\right)^{2}}, \\
& K_{3}=\frac{1}{\omega_{2}^{2}-\left(\omega_{1}+\omega_{2}\right)^{2}}, \quad K_{4}=\frac{1}{\omega_{2}^{2}-\left(\omega_{2}-\omega_{1}\right)^{2}}, \\
& K_{5}=\frac{1}{\omega_{2}^{2}-\left(\omega_{3}-\omega_{1}\right)^{2}}, \quad K_{6}=\frac{1}{\omega_{3}^{2}-\left(\omega_{1}+\omega_{2}\right)^{2}},
\end{aligned}
$$$$
K_{7}=\frac{1}{\omega_{3}^{2}-\left(\omega_{1}+\omega_{3}\right)^{2}} .
$$

Coefficients $b_{i}$ of Eqs. (10) are

$$
\begin{aligned}
& b_{1}=8 \alpha_{1} \alpha_{4}-4 \alpha_{2} \alpha_{5}+k_{1}, \quad b_{2}=8 \alpha_{1} \alpha_{5}+4 \alpha_{2} \alpha_{3}+k_{3}, \\
& b_{3}=-2 \alpha_{1} \sigma_{1}, \quad b_{4}=-4 \alpha_{2} \alpha_{4}+k_{2}, \\
& b_{5}=-2 \alpha_{2} \sigma_{1}, \quad b_{6}=4 \alpha_{1} \alpha_{3}+k_{5}, \\
& b_{7}=4 \alpha_{2} \alpha_{3}+k_{7}, \quad b_{8}=4 \alpha_{3} \alpha_{4}+k_{8}, \\
& b_{9}=4 \alpha_{3} \alpha_{5}+k_{6}, \quad b_{10}=k_{4}, \quad b_{11}=2 \alpha_{3} \sigma_{1}, \\
& b_{12}=-4 \alpha_{1} \alpha_{4}+4 \alpha_{2} \alpha_{5}+k_{13}, \quad b_{13}=-4 \alpha_{2} \alpha_{4}+k_{12}, \\
& b_{14}=4 \alpha_{3} \alpha_{4}-4 \alpha_{5}^{2}+k_{11}, \quad b_{15}=-2 \alpha_{4} \sigma_{1}, \\
& b_{16}=4 \alpha_{1} \alpha_{5}+k_{9}, \quad b_{17}=-4 \alpha_{4} \alpha_{5}+k_{10}, \\
& b_{18}=2 \sigma_{1} \alpha_{5},
\end{aligned}
$$

where $k_{i}$ are

$$
\begin{aligned}
& k_{1}=c_{6}+2 c_{1} c_{8} K_{5}+c_{3} c_{7} K_{4}, \\
& k_{2}=2 c_{4}+2 c_{1} c_{7} K_{3}+2 c_{1} c_{7} K_{4}+c_{2} c_{15} K_{6}, \\
& k_{3}=2 c_{5}+2 c_{2} c_{16} K_{7}+c_{3} c_{8} K_{5}, \\
& k_{4}=3 c_{9}+2 \frac{c_{7} c_{1}}{\omega_{1}^{2}}+c_{7} c_{1} K_{1}, \quad k_{5}=c_{10}, \\
& k_{6}=c_{12}+c_{7} c_{8} K_{5}, \quad k_{7}=2 c_{13}+2 \frac{c_{7} c_{2}}{\omega_{1}^{2}}+c_{8} c_{3} K_{2}, \\
& k_{8}=2 c_{19}+c_{7}^{2} K_{3}+c_{7}^{2} K_{4}+c_{8} c_{15} K_{6},
\end{aligned}
$$

$k_{9}=3 c_{18}+2 \frac{c_{16} c_{2}}{\omega_{1}^{2}}$

$$
\begin{aligned}
& k_{10}=c_{19}+c_{15} c_{7} K_{4}, \quad k_{11}=2 c_{20}+c_{15} c_{8} K_{5}+c_{16}^{2} K_{7}, \\
& k_{12}=2 c_{22}+c_{15} c_{3} K_{2}+2 \frac{c_{16} c_{1}}{\omega_{1}^{2}}, \quad k_{13}=c_{21} .
\end{aligned}
$$

Coefficients $h_{i}, d_{1,2,3}^{*}$ and $f_{1}^{*}$ of Eqs. (14) are

$$
\begin{aligned}
& h_{1}=\alpha_{1}+\frac{b_{3}}{4 \omega_{1}}, \quad h_{2}=\alpha_{2}+\frac{b_{5}}{4 \omega_{1}}, \quad h_{3}=\frac{b_{1}}{8 \omega_{1}}, \\
& h_{4}=\alpha_{3}+\frac{b_{11}}{4 \omega_{2}}, \quad h_{5}=\frac{b_{6}}{8 \omega_{2}}, \quad h_{6}=\frac{b_{9}}{8 \omega_{2}}, \\
& h_{7}=\alpha_{5}+\frac{b_{18}}{4 \omega_{3}}, \quad h_{8}=\alpha_{4}+\frac{b_{15}}{4 \omega_{3}}, \quad h_{9}=\frac{b_{12}}{8 \omega_{3}}, \\
& h_{10}=\frac{b_{17}}{8 \omega_{3}}, \quad h_{11}=\frac{b_{13}}{8 \omega_{3}}, \quad h_{12}=\frac{b_{14}}{8 \omega_{3}}, \\
& h_{13}=\frac{b_{16}}{8 \omega_{3}}, \quad h_{14}=\frac{b_{2}}{8 \omega_{1}}, \quad h_{15}=\frac{b_{4}}{8 \omega_{1}}, \\
& h_{16}=\frac{b_{7}}{8 \omega_{2}}, \quad h_{17}=\frac{b_{8}}{8 \omega_{2}}, \quad h_{18}=\frac{b_{10}}{8 \omega_{2}}, \\
& d_{1}^{*}=\frac{d_{1}}{2}, \quad d_{2}^{*}=\frac{d_{2}}{2}, \quad d_{3}^{*}=\frac{d_{3}}{2}, \quad f_{1}^{*}=\frac{f_{1}}{\omega_{1}} .
\end{aligned}
$$

\section{References}

[1] A. Di Egidio, A. Luongo, F. Vestroni, A non-linear model for the dynamics of open cross-section thin-walled beamsPart I: formulation, Int. J. Non-linear Mech. 37 (2003) 1067-1081.

[2] M.R.M. Crespo da Silva, C.C. Glynn, Nonlinear flexural-flexural-torsional dynamics of inextensional beams. II: forced motion, J. Struct. Mech. 6 (1) (1978) 437-448.

[3] F. Pernjin, H. Nayfeh Ali, Non-linear non-planar oscillations of a cantilever beam under lateral base excitations, Int. J. Non-Linear Mech. 25 (5) (1990) 455-474.

[4] A. Luongo, G. Rega, F. Vestroni, Non resonant non-planar free motions of inextensional non-compact beams, J. Sound Vib. 134 (1) (1989) 73-86.

[5] M.R.M. Crespo da Silva, C.L. Zaretzky, Nonlinear flexuralflexural-torsional interaction in beams including the effect of torsional dynamics. I: primary resonance, Nonlinear Dyn. 5 (1994) 3-23.

[6] M.R.M. Crespo da Silva, C.L. Zaretzky, Nonlinear flexuralflexural-torsional interaction in beams including the effect 
of torsional dynamics. II: combination resonance, Nonlinear Dyn. 5 (1994) 24-42.

[7] F. Benedettini, A. Di Egidio, Theoretical and experimental finite forced dynamics of a cantilever beam: dynamic instability and modal coupling, Proceedings of DECT99, September 14-17, Las Vegas, Nevada, 1999.
[8] H. Nayfeh Ali, Perturbation Methods, Pure \& Applied Mathematics-A Wiley Interscience Series of Text, Monographs \& Tracts, New York, 1973.

[9] H. Nayfeh Ali, Topical course on nonlinear dynamics, in: Perturbation Methods in Nonlinear Dynamics, Società Italiana di Fisica, Santa Margherita di Pula, Sardinia, 1985. 